\title{
Acute aortic dissection presenting as status epilepticus
}

\author{
Rachel Eikelboom MD, Noam Katz MD MSc
}

Cite as: CMAJ 2020 March 16;192:E283-5. doi: 10.1503/cmaj.190822

A 57-year-old woman with no history of seizure was brought into the emergency department in status epilepticus. Her only medical history was hypothyroidism and her only prescription medication was levothyroxine.

The patient had been feeling well and participating in physiotherapy for an ankle injury when she collapsed suddenly. She had generalized tonic-clonic movements for about 15 minutes, which were witnessed. Upon arrival, paramedics confirmed generalized tonic-clonic movements and terminated them successfully with intravenous midazolam.

Upon arrival at the emergency department, the patient was unresponsive. Her vital signs were heart rate 74 beats/min, blood pressure $87 / 53 \mathrm{~mm} \mathrm{Hg}$, oxygen saturation $95 \%$ on $10 \mathrm{~L}$ of supplemental oxygen and temperature $37.4^{\circ} \mathrm{C}$. A neurologic examination of the patient showed left-sided rigidity with the left arm hyperflexed at the elbow and left leg hyperextended at the hip, with upgoing toes. Her pedal pulses were palpable and equal bilaterally; an upper limb vascular examination was not documented. After 10 minutes of treatment with increasing doses of intravenous midazolam because of concerns about nonconvulsive status epilepticus, the patient's neurologic status did not improve, and she was intubated and sedated with propofol. A norepinephrine infusion was started before intubation and rapidly uptitrated because of worsening hypotension.

The results of a number of investigations were available within 1 hour of presentation to the emergency department. Her complete blood count, extended electrolytes, fingerstick glucose, high-sensitivity troponin T, limited toxicology screen and uninfused brain computed tomography (CT) were within normal limits. An electrocardiogram showed normal sinus rhythm. Chest radiography did not show widening of the mediastinum. As a result of persistent hypotension, a rapid ultrasound for shock and hypotension (RUSH) examination was performed using point-of-care ultrasound. This showed a dilated ascending aorta on the parasternal long axis and short axis views (Figure 1 and Figure 2) and an intimal dissection flap beginning in the aortic root, which extended into the abdominal aorta, prompting an emergent consult to the on-call cardiac surgeon.

A contrast-enhanced biphasic CT angiogram of the patient's chest and abdomen was performed within 2 hours of presentation to the emergency department. It showed a type A aortic dissection extending from the coronary sinuses to the right common iliac artery (Figure 3). The aortic valve was tri-leaflet but the

\section{KEY POINTS}

- Thirty percent of acute type A aortic dissections present with neurologic symptoms, but seizures are uncommon.

- The combination of hypotension with neurologic deficits should trigger physicians to consider acute aortic dissection higher in the differential diagnosis for shock.

- The rapid ultrasound for shock and hypotension (RUSH) examination provides a systematic ultrasonographic examination for patients in shock, and is particularly useful in differentiating cardiogenic and hypovolemic shock.

- In accordance with the Canadian Cardiovascular Society position statement on thoracic aortic disease, first-degree relatives of patients with thoracic aortic aneurysms or aortic dissections should be screened with echocardiography.

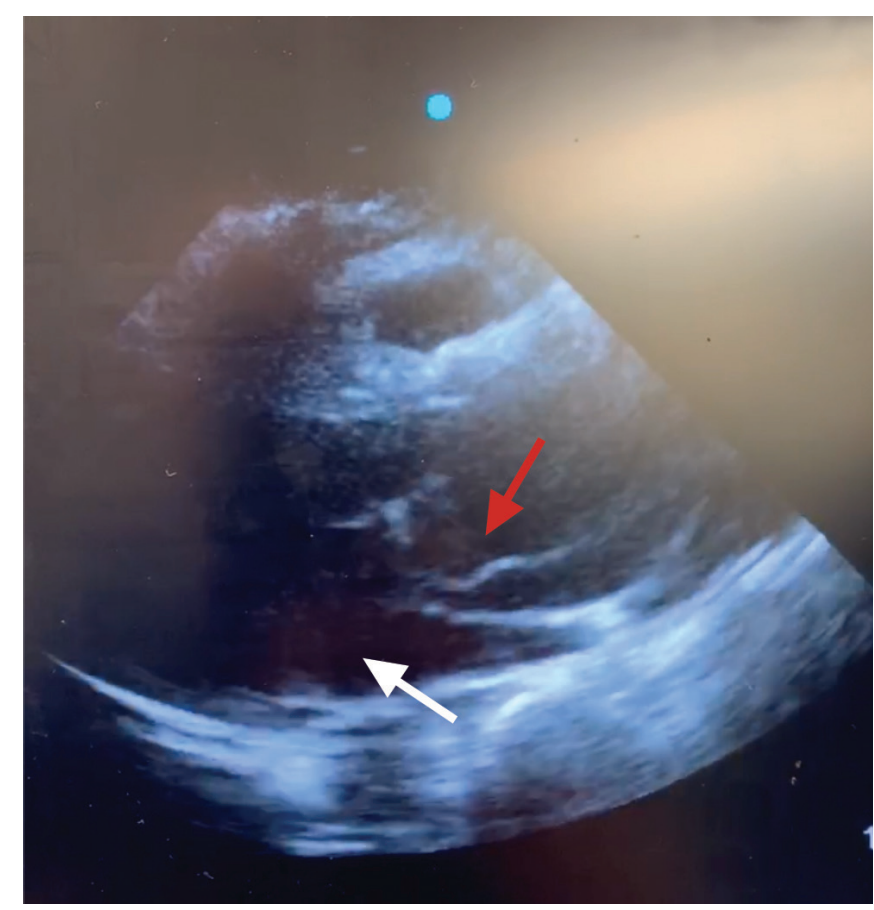

Figure 1: Transthoracic echocardiogram of a 57-year-old woman (parasternal long axis) showing dissection flap in aortic root (red arrow). The white arrow indicates the left ventricle. 


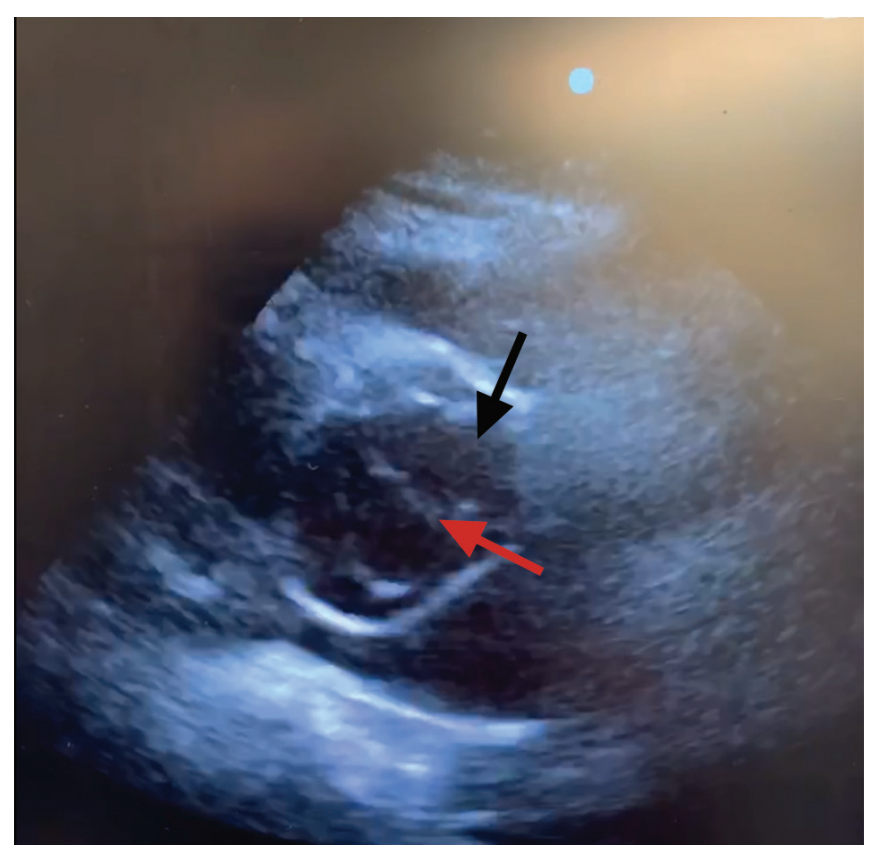

Figure 2: Transthoracic echocardiogram (parasternal short axis) showing dissection flap (red arrow) in aortic root (black arrow).

ascending aorta was aneurysmal, measuring $55 \mathrm{~mm}$ at the sinotubular junction. The right common carotid artery was occluded, accounting for the patient's neurologic status. The left renal artery originated from the false lumen; the remaining mesenteric vessels were perfused by the true aortic lumen.

The patient was taken directly to the operating room. Her intraoperative transesophageal echocardiogram (Appendix 1, available at www.cmaj.ca/lookup/suppl/doi:10.1503/cmaj.190822/-/DC1)

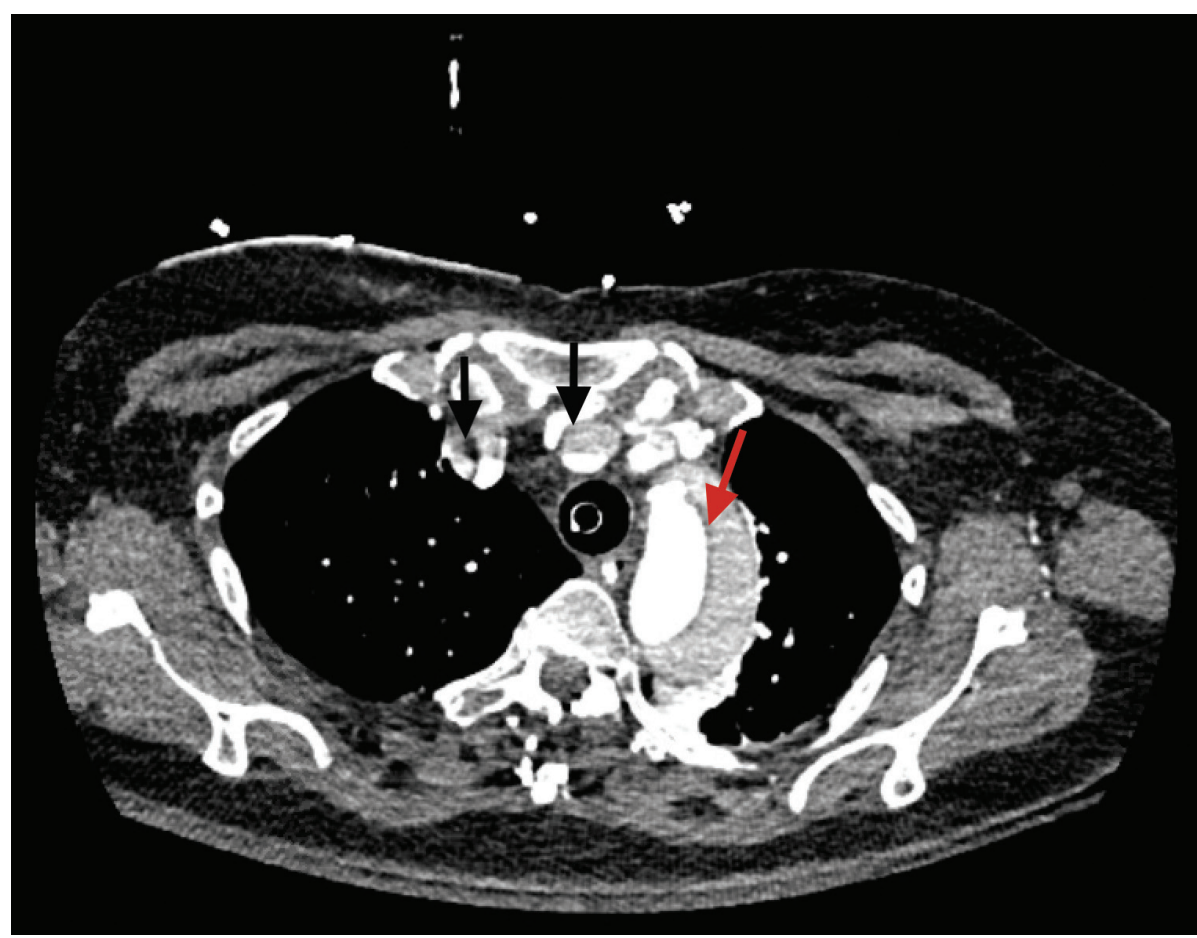

Figure 3: Infused computed tomography scan of the chest showing dissection flap (red arrow) in aortic root and malperfusion of right common carotid and subclavian arteries (black arrows). showed severe aortic insufficiency. Cardiopulmonary bypass was started through the left femoral artery and vein. She underwent a Bentall procedure with tissue aortic valve replacement and hemiarch replacement under hypothermic circulatory arrest with partial antegrade cerebral perfusion through the left common carotid artery.

The patient was extubated on the first postoperative day with intact neurologic status and was transferred out of the cardiac surgery intensive care unit the next day. There was no evidence of limb ischemia and no impairment in renal function. She was discharged on the ninth postoperative day. Given that the patient's mother and aunt had died of ruptured aortic aneurysms, the patient was referred to a medical geneticist for further investigation. It was recommended that first-degree family members be screened for aortic aneurysm with echocardiography.

\section{Discussion}

Acute aortic dissection classically presents with sudden chest or back pain, which is maximal at onset. Classic physical examination findings include differential blood pressures and pulses between right and left limbs. The triad of sudden pain, differential blood pressure or pedal pulses and mediastinal widening on chest radiography has a $96 \%$ positive predictive value for acute aortic dissection. ${ }^{1}$

Although specific for aortic dissection, the absence of these findings on physical examination does not rule out the diagnosis. In a case series of 102 patients with acute aortic dissection, only $51 \%$ presented with the classic ripping chest pain, and only $38 \%$ had pulse or blood pressure differentials. ${ }^{2}$ The diagnosis of acute aortic dissection is initially missed in $38 \%$ of patients, and up to $28 \%$ of patients first receive the diagnosis at postmortem examination. ${ }^{2}$

In addition to the classic findings, acute aortic dissection can cause malperfusion of any organ system, leading to wide-ranging signs and symptoms of endorgan dysfunction. As many as $30 \%$ of patients present with neurologic symptoms, caused either by hypotension or by dissection of the vessels perfusing the brain. $^{2}$ Stroke $(5 \%-10 \%)$ and syncope $(12 \%)$ are the most common neurologic symptoms. The incidence of seizure in acute type $A$ dissection is incompletely described in the literature, but in small case series is reported in $3 \%$ of cases. ${ }^{2,3}$

Given that acute type A aortic dissection is universally fatal without expeditious surgical intervention, clinicians must maintain a high index of suspicion in patients presenting to the emergency department with unexplained neurologic symptoms, particularly in the presence of a family history of aortic aneurysm or aortic dissection. 


\section{Point-of-care ultrasound and acute aortic dissection}

Point-of-care ultrasound can assist in expeditious diagnosis of acute aortic dissection in the emergency department. The presence of an aortic intimal dissection flap on transthoracic echocardiography establishes the diagnosis definitively. Other findings consistent with acute aortic dissection include pericardial effusion and dilation of the aortic outflow tract. ${ }^{4}$ When performed by experienced operators, the sensitivity of point-of-care ultrasound is $88 \%-96 \%$ for acute type A aortic dissections. ${ }^{4,5}$ Importantly, clinicians can use point-of-care ultrasound to reduce time to diagnosis and can arrange for portable transesophageal echocardiography in the emergency department. Transesophageal echocardiography is the gold standard for diagnosis of acute aortic dissection in patients who are too unstable to undergo CT angiography, which is the imaging modality of choice for stable patients. ${ }^{6}$

\section{Rapid ultrasound for shock and hypotension examination}

The RUSH examination is a 3-part ultrasound assessment of undifferentiated shock and hypotension. The "pump" (cardiac function), "tank" (volume status) and "pipes" (arteries and veins) are systematically examined.

Cardiac function is assessed with standard parasternal long and short axis, subxiphoid and apical 4-chamber views. Pericardial effusion, impaired left ventricular systolic function and right ventricular strain or dilation should be ruled out. Intravascular volume status is assessed by visualizing the inferior vena cava, although the reliability of this assessment has been contested. Extravascular volume accumulation in the lungs and abdomen is assessed with lung, hepatorenal, splenorenal and bladder views. The thoracic and abdominal aorta are visualized for dissection and aneurysm rupture. If desired, the deep leg veins can be scanned to look for deep vein thrombosis. ${ }^{7}$

A systematic review and meta-analysis of the accuracy of the RUSH examination in differentiating shock subtype was recently reported in the Canadian Journal of Emergency Medicine. ${ }^{8}$ Although only 4 studies involving 357 patients were identified, the results were encouraging, with sensitivity exceeding $80 \%$ and specificity exceeding $95 \%$ for most shock subtypes. Diagnostic accuracy was particularly high in cardiogenic (sensitivity $83 \%$, specificity $97 \%$ ) and obstructive (sensitivity $93 \%$, specificity $98 \%$ ) shock. ${ }^{8}$ The utility of the RUSH examination in diagnosing cause of shock is incompletely described.

In our case, we performed the RUSH examination because of persistent hypotension that was incongruous with the patient's seizures, leading us to a diagnosis of acute aortic dissection. Thus, our experience supports the use of an organized and systematic approach like the RUSH examination for critically ill patients with undifferentiated shock.

\section{Screening for thoracic aortic aneurysms}

The Canadian Cardiovascular Society recommends that first-degree family members of patients with thoracic aortic disease are screened once for aortic aneurysm with transthoracic echocardiography.
Discovery of aortic dilation or aneurysm should prompt referral for cardiac or vascular surgery consultation. Patients with a strong family history of thoracic aortic aneurysms should be referred for genetic workup. ${ }^{9}$ Appropriate screening and intervention can prevent life-threatening complications such as acute aortic dissection. In the case presented here, the patient had a strong family history of aortic dissection, which was unknown to the emergency department team and for which she had not been screened.

\section{Conclusion}

Acute type A aortic dissection is universally fatal without expeditious surgical intervention. Rapid diagnosis can significantly reduce major morbidity and mortality. Fewer than half of patients present with the classic triad of chest pain, differential blood pressures or pulses, and widening of the mediastinum on chest radiography.

\section{References}

1. Ohle R, Kareemi HK, Wells G, et al. Clinical examination for acute aortic dissection: a systematic review and meta-analysis. Acad Emerg Med 2018;25:397-412.

2. Khan IA, Nair CK. Clinical, diagnostic, and management perspectives of aortic dissection. Chest 2002;122:311-28.

3. Gaul C, Dietrich W, Friedrich I, et al. Neurological symptoms in type A aortic dissections. Stroke 2007;38:292-7.

4. Gibbons R, Smith D, Mulflur M, et al. 364 point-of-care ultrasound for the detection of aortic dissections in the emergency department. Ann Emerg Med 2017;70(Suppl):S143. doi: 10.1016/j.annemergmed.2017.07.334.

5. Nazerian P, Vanni S, Castelli M, et al. Diagnostic performance of emergency transthoracic focus cardiac ultrasound in suspected acute type A aortic dissection. Intern Emerg Med 2014;9:665-70.

6. Pare JR, Liu R, Moore CL, et al. Emergency physician focused cardiac ultrasound improves diagnosis of ascending aortic dissection. Am J Emerg Med 2016;34:486-92.

7. Seif $D$, Perera $P$, Mailhot $T$, et al. Bedside ultrasound in resuscitation and the rapid ultrasound in shock protocol. Crit Care Res Pract 2012;2012:503254.

8. Stickles SP, Carpenter CR, Gekle R, et al. The diagnostic accuracy of a point-ofcare ultrasound protocol for shock etiology: a systematic review and metaanalysis. CJEM 2019;21:406-17.

9. Boodhwani M, Andelfinger G, Leipsic J, et al.; Canadian Cardiovascular Society. Canadian Cardiovascular Society position statement on the management of thoracic aortic disease. Can J Cardiol 2014;30:577-89.

Competing interests: Noam Katz reports receiving instructor fees for CPOCUS IP-Core and IP-Resus courses, which include obtaining cardiac views, and for an Echo-Guided Life Support course, which focuses on bedside echocardiography. No other competing interests were declared.

This article has been peer reviewed.

The authors have obtained patient consent.

Affiliations: Cardiac Surgery (Eikelboom), St. Boniface Hospital; Faculty of Medicine (Eikelboom), University of Manitoba; Emergency Department (Katz), St. Boniface Hospital; Department of Emergency Medicine (Katz), Winnipeg, Man.

Contributors: Rachel Eikelboom wrote the first draft of the manuscript. Both authors contributed to the conception and design of the work, revised the manuscript critically for important intellectual content, gave final approval of the version to be published and agreed to be accountable for all aspects of the work.

Correspondence to: Rachel Eikelboom, eikelbor@myumanitoba.ca 